\title{
Aimants permanents FeNdB, obtenus à partir de la solidification rapide influencé du vanadium
}

\author{
A. TANGUY, D. BINESTI, J. BRAS*, J. DEGAUQUE*, M. FAGOT*, A. CHAMBEROD** et \\ F. VANONI*
}

EDF - DER, 1 av du Géneral de Gaulle, 92141 Clamart, France

"INSA, LPS Toulouse, Dpt Physique, av de Rangueil, 31077 Toulouse, France

${ }^{*}$ CENG-DRFMC, Av des Martyrs, 38041 Grenoble, France

\begin{abstract}
Résumé: L'influence d'ajouts de vanadium $(1 ; 1,6 ; 2,1 ; 2,3 \%$ at) sur les propriétés des matériaux rapidement solidifiès est étudiée ici. Ces additions provoquent une diminution de la température de Curie, tandis que la cristallisation de la phase amorphe contenue dans les rubans est retardée. Pour l'instant aucune évolution microstructurale n'a pu être décelée. La coercitivité des aimants de type l est maximale pour $1 \%$ d'ajout. Pour les aimants de type II et au delà de 1,6\%, l'ajout de vanadium s'accompagne d'un accroissement de la coercitivité et d'une modification des mécanismes d'aimantation.
\end{abstract}

\begin{abstract}
The influence of vanadium additions $(1 ; 1,6 ; 2,1 ; 2,3 \%$ at) on the properties of melt spun materials is studied. These additions lower the Curie temperature, while the cristallisation of the amorphous phase present in ribbons is delayed. No evolution of the microstructure was observed at this time. For type I magnets, coercivity is maximum for a $1 \%$ addition, for type $I$ and above $1,6 \%$, vanadium addition induces an increase in coercivity and a change in magnetisation mechanisms.
\end{abstract}

\section{INTRODUCTION:}

Le développement d'aimants terres rares à base de fer peut contribuer à la mise en place de moteurs électriques performants, dans des conditions économiques acceptables.

Les aimants fer néodyme bore, dont l'énergie spécifique à température ambiante reste inégalée, sont desservis par un médiocre comportement en température et une forte sensibilité à la corrosion.

L'introduction de vanadium dans la composition d'aimants frittés a toutefois permis des progrès significatifs dans ces deux directions [1].

L'effet des ajouts de vanadium dans des matériaux solidifiès rapidement, a priori moins sensibles à la corrosion, est étudié ici. 
PARTIE EXPERIMENTALE

Les matériaux sont élaborés à partir d'une composition témoin exempte de vanadium :

(t): $\mathrm{Fe}_{80,1} \mathrm{Nd}_{13,9} \mathrm{~B}_{5,4} \mathrm{Al}_{0,6}$

Cet alliage représente le meilleur compromis coercitivité / rémanence pour des aimants compressés à chaud (aimants de type II)[2]. II peut être décomposé en deux termes: la phase magnétique dure et un ajout binaire: $(0,4 \mathrm{Nd}+0,6 \mathrm{Fe})$. Dans le but de former des précipités $\mathrm{FeV}_{2} \mathrm{~B}_{2}$ obtenus dans le cas des aimants frittés, le vanadium a été introduit dans l'ajout dont la composition type est alors: $(1-x)(0,4 \mathrm{Nd}+0,6 \mathrm{Fe})+x(0,2 \mathrm{Fe}+0,4 \mathrm{~V}+0,4 \mathrm{~B})$ ce qui conduit à la formule générale:

$$
\mathrm{Fe}_{80,1-y} \mathrm{Nd}_{13,9-y} \mathrm{~B}_{5,4+y} \mathrm{~V}_{y} \mathrm{Al}_{0,6}
$$

avec $y=1 ; 1,6 ; 2,1$ et 2,3 valeurs qui correspondent respectivement aux compositions dites (1), (2), (3) et (4).

Les alliages sont préparés à partir de matériaux de pureté $3 \mathrm{~N}$, puis trempés sur roue avec une vitesse linéaire de $23 \mathrm{~m} / \mathrm{s}$. Deux types d'aimants sont rẻalisés à partir des rubans broyés:

- type I : obtenu par compactage à froid dans une résine

- type II : obtenu par compression à chaud avec ou sans liant métallique

La température de Curie $\left(T_{c}\right)$ et la température de cristallisation $\left(T_{x}\right)$ de la phase amorphe présente dans les rubans sont mesurées par analyse enthalpique différentielle avec une vitesse de montée de $40^{\circ} \mathrm{C} / \mathrm{mn}$ (Perkin Elmer, D.S.C.7).

La microstructure est observée en microscopie électronique en transmission (JEOL 200CX) couplè à un analyseur X (sonde EDAX).

Les propriétés magnétiques sont mesurées avec un hystérésigraphe (LDJ,5500H) associé à un banc d'aimantation fournissant des impulsions de 10 Tesla.

RESULTATS ET DISCUSSION

\section{A.ETUDE DES RUBANS:}

\section{Analyse EnthalpiQue Differentielle:}

La chute de $T_{c}$ dès la composition (1), puis sa stabilisation pour les teneurs supérieures en vanadium (cf. tableau I), semble indiquer que l'on atteint la limite de solubilité du vanadium dans la phase magnétique $\mathrm{Nd}_{2} \mathrm{Fe}_{14} \mathrm{~B}$ dès $1 \%$ d'ajout. En revanche, la température de cristallisation augmente de façon continue. La cristallisation pourrait être retardée par une ségrégation du vanadium aux joints de grains.

tableau I: évolution de $T_{C}$ et de $T_{X}$ avec la teneur en vanadium.

\begin{tabular}{|c|c|c|c|c|c|}
\hline composition & $(\mathrm{t})$ & $(1)$ & $(2)$ & $(3)$ & (4) \\
\hline $\mathrm{T}_{\mathrm{c}}\left({ }^{\circ} \mathrm{C}\right)$ & 309 & 304 & 304 & 303 & 305 \\
\hline $\mathrm{T}_{\mathrm{X}}\left({ }^{\circ} \mathrm{C}\right)$ & 600 & 604 & 609 & 613 & \\
\hline
\end{tabular}




\section{MICROSCOPIE ELECTRONIQUE EN TRANSMISSION:}

Les rubans bruts de trempe ont des tailles de grains comprises entre 20 et $300 \mathrm{~nm}$. Aucun agglomérat ni précipité riche en vanadium n'a été décelé. Par contre, les rubans recuits $10 \mathrm{mn}$ à $620^{\circ} \mathrm{C}$ contiennent de petits précipités $(10$ à $20 \mathrm{~nm})$ de morphologie identique à celle des précipités $\mathrm{FeV}_{2} \mathrm{~B}_{2}$ observés dans le cas des aimants frittés avec vanadium [3]. Leur faible taille n'a pas permis d'en déterminer la composition.

\section{B.Aimants DE TYPE I:}

\section{EFFET DU BROYAGE:}

Afin d'étudier l'influence de la taille des poudres sur les propriétés magnétiques (et sur un éventuel effet d'anisotropie) les rubans ont été broyés et répartis en trois granulométries (థ) différentes: $\Phi<40 \mu \mathrm{m}, 100 \mu \mathrm{m}<\Phi<200 \mu \mathrm{m}$ et $\Phi>200 \mu \mathrm{m}$. Le broyage a été réalisé d'une part à l'air, d'autre part sous atmosphère d'argon sec.

Dans le premier cas, on observe une forte chute de la coercitivité et de l'énergie spécifique pour les granulométries inférieures à $40 \mu \mathrm{m}$. Cet effet semble du à la présence de phases douces résultant de l'oxydation de la phase magnétique (tableau II et fig.1), phénomène plus marqué dans le cas des faibles granulométries où le rapport surface sur volume est plus important. La présence d'une plus forte concentration d'oxygène pour les poudres de faible granulométrie a été confirmée par des mesures de taux d'oxygène. Dans le cas d'un broyage sous argon, les propriètés sont conservées pour les faibles granulométries (tableau II).

tableau II: influence sur les propriétés magnétiques de l'atmosphère de broyage

\begin{tabular}{|c|c|c|}
\hline & $\mathrm{H}^{*}$ & $\mathrm{BH}^{*}$ \\
\hline broyé à I'air & $44 \%$ & $39 \%$ \\
\hline broyé sous Ar & $100 \%$ & $100 \%$ \\
\hline
\end{tabular}

nota:

$$
\begin{aligned}
& H^{*}=H c_{j(\Phi<40 \mu m)} / H c_{j(\Phi>200 \mu m)} \\
& (B H)^{*}=(B H) \max (\Phi<40 \mu m) /(B H) \max (\Phi>200 \mu m)
\end{aligned}
$$

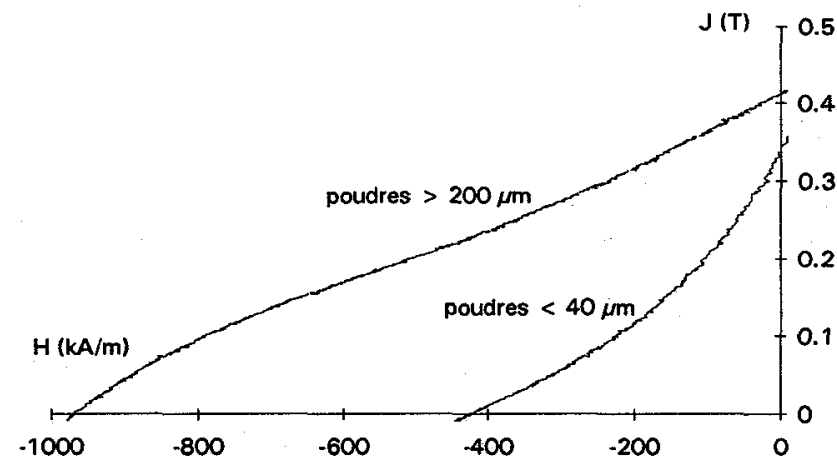

figure 1:

courbes de désaimantation d'aimants de type I réalisés à partir de poudres broyées à l'air 


\section{EFFET DE LA PRESSION:}

La densité, et donc la rémanence sont peu influencées par l'augmentation de pression de $65 \mathrm{MPa}$ à $200 \mathrm{MPa}$ (tableau III). Le champ coercitif n'est pas modifié.

tableau III: effets de la pression sur les propriétés d'aimants de composition (t).

\begin{tabular}{|c|c|c|}
\hline Pression & $65 \mathrm{MPa}$ & $200 \mathrm{MPa}$ \\
\hline densité $\left(\mathrm{g} / \mathrm{cm}^{3}\right)$ & 5,54 & 5,66 \\
\hline $\mathrm{B}_{\mathrm{r}}(\mathrm{T})$ & 0,42 & 0,50 \\
\hline
\end{tabular}

\section{EFFET DU VANADIUM:}

Les résultats ne montrent pas de variation significative de la rémanence qui vaut en moyenne $0,45 \mathrm{~T}( \pm 0,05 \mathrm{~T})$. La coercitivité est maximale pour une teneur en vanadium de $1 \%$ (composition (1), tableau (V).

tableau IV: variations de $\mathrm{Hc}_{\mathrm{j}}$ en fonction de la teneur en vanadium.

\begin{tabular}{|c|c|c|c|c|}
\hline composition & $(\mathrm{t})$ & $(1)$ & $(2)$ & $(3)$ \\
\hline $\mathrm{Hc}_{j}(\mathrm{kA} / \mathrm{m})$ & 950 & 1050 & 850 & 800 \\
\hline
\end{tabular}

\section{C.AIMANTS de TYPE II:}

Les compressions sont réalisées à $620^{\circ} \mathrm{C}, 720^{\circ} \mathrm{C}$ et $750^{\circ} \mathrm{C}$ sous une pression de $380 \mathrm{MPa}$. Les mesures sont regroupées dans le tableau $\mathrm{V}$, ci dessous.

\begin{tabular}{|c|c|c|c|c|}
\hline $\begin{array}{c}\text { Température } \\
\left({ }^{\circ} \mathrm{C}\right)\end{array}$ & composition & $\begin{array}{c}\text { densité } \\
\left(\mathrm{g} / \mathrm{cm}^{3}\right)\end{array}$ & $\begin{array}{c}\mathrm{B}_{\mathbf{r}} \\
(\mathrm{T})\end{array}$ & $\begin{array}{c}\mathrm{Hc}_{\mathrm{j}} \\
(\mathrm{kA} / \mathrm{m})\end{array}$ \\
\hline \multirow{3}{*}{620} & $(\mathrm{t})$ & 7,1 & 0.75 & 790 \\
& $(2)$ & 5,7 & 0,55 & 860 \\
& $(3)$ & 0,64 & 1000 \\
\hline 720 & $(\mathrm{t})$ & 7,4 & 0,78 & 900 \\
& $(1)$ & 7,35 & 0,69 & 820 \\
& $(2)$ & 7,25 & 0,68 & 660 \\
& $(3)$ & 7,1 & 0,68 & 660 \\
\hline 750 & $(\mathrm{t})$ & 7,4 & 0,70 & 660 \\
& $(1)$ & 7,4 & 0,72 & 660 \\
& $(2)$ & 7,4 & 0,73 & 680 \\
& $(3)$ & 7,4 & 0,67 & 650 \\
\hline
\end{tabular}


A $620^{\circ} \mathrm{C}$, les aimants contenant du vanadium sont fragiles et peu denses. Le vanadium augmente le champ coercitif et modifie les mécanismes d'aimantation comme l'indiquent les figures 2 et 3 :

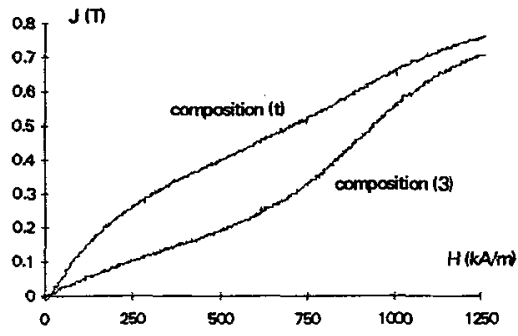

figure 2

courbes de première aimantation d'aimants de type II réalisés à $620^{\circ} \mathrm{C}$

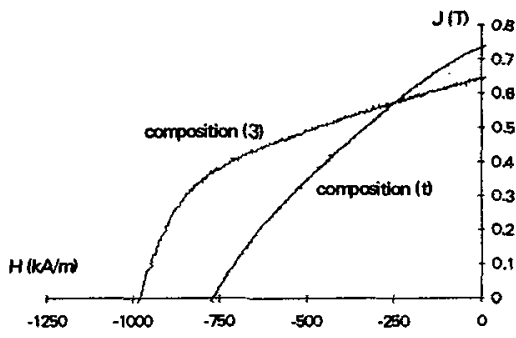

figure 3:

courbes de désaimantation d'aimants de type II réalisés à $620^{\circ} \mathrm{C}$

Seule la température de $750^{\circ} \mathrm{C}$ apporte une densification complète $1100 \%$ de la densitè théoriquel, mais au détriment de la coercitivité. $L$ 'effet bénéfique du vanadium est annihilé.

Afin d'obtenir, dès $620^{\circ} \mathrm{C}$, des aimants de bonne tenue mécanique, deux liants métalliques, du cuivre ou du cobalt en poudre, ont été ajoutés aux rubans broyés.

Les résultats sont regroupés dans le tableau VI suivant:

\begin{tabular}{|c|c|c|c|c|}
\hline $\begin{array}{c}\text { ajout } \\
\text { (\% pds) }\end{array}$ & composition & $\begin{array}{c}\text { densité } \\
\left(\mathrm{g} / \mathrm{cm}^{3}\right)\end{array}$ & $\begin{array}{c}\mathrm{B}_{\mathrm{r}} \\
(\mathrm{T})\end{array}$ & $\begin{array}{c}\mathrm{Hc}_{\mathrm{j}} \\
(\mathrm{kA} / \mathrm{m})\end{array}$ \\
\hline néant & $(\mathrm{t})$ & 7,55 & 0,74 & 780 \\
\hline $10 \% \mathrm{Co}$ & $(\mathrm{t})$ & 7,44 & 0,70 & 590 \\
& $(4)$ & 6,75 & 0,60 & 775 \\
\hline $10 \% \mathrm{Cu}$ & $(4)$ & 7,55 & 0,64 & 560 \\
\hline $2 \% \mathrm{Co}$ & $(\mathrm{t})$ & 7,57 & 0,74 & 715 \\
& $(4)$ & 7,21 & 0,71 & 955 \\
\hline $2 \% \mathrm{Cu}$ & $(\mathrm{t})$ & 7.57 & 0,72 & 760 \\
& $(4)$ & 7,09 & 0,69 & 980 \\
\hline
\end{tabular}

L'ajout de $2 \%$ de liant présente un bon compromis propriétés magnétiques/tenue mécanique. On retrouve, en particulier, l'effet bénéfique du vanadium sur la coercitivité, observé en l'absence de liant (tableau V).

Les résultats obtenus pour la composition témoin avec ou sans liant sont semblables. Le cuivre et le cobalt n'ont pas ou peu diffusé dans la phase $\mathrm{Nd}_{2} \mathrm{Fe}_{14} \mathrm{~B}$ puisque, dans le cas contraire, il en aurait résulté une chute de coercitivité dans le cas du cobalt [4] et un accroissement dans le cas du cuivre [5]. Les propriétés magnétiques sont peu influencées par la faible quantité de liant présent entre les morceaux de rubans.

Par contre avec $10 \%$ d'ajout, le liant présent en quantité plus importante apporte une meilleure cohésion mécanique, mais fait chuter la coercitivité (tableau V).

Plusieurs mécanismes peuvent être mis en cause. Le cobalt engendre la présence de phases douces (cobalt ou composés intermétalliques avec les autres constituants), le cuivre, non magnétique, peut créer des zones de germination de domaines inverses annulant l'effet bénéfique de découplage que l'on aurait pu attendre; la formation de composés intermétalliques est aussi envisageable.

Le rôle exact de ces liants reste à confirmer. 


\section{CONCLUSIONS}

Aimants de type I: l'optimum est obtenu pour $1 \%$ d'ajout de vanadium. Dans le cas d'un broyage à l'air, les propriétés sont fortement affectées par l'emploi de poudres de faible granulométrie $(\Phi<40 \mu \mathrm{m})$ trop sensibles à l'oxydation.

Aimants de type II: pour une température de $620^{\circ} \mathrm{C}$, la présence de vanadium augmente la coercitivité mais fragilise les aimants. Un bon compromis a èté obtenu par un ajout de $2 \%$ en poids de cobalt ou de cuivre.

Nous remercions la société Ugimag pour les mesures de taux d'oxygène.

\section{Références:}

[1]:P. Tenaud, F.Vial, conf symposium SEE Grenoble, 13-15 juin 90

[2]:A. Chamberod, F. Vanoni, Concerted Europeen Action on Magnets (CEAM) ELSEVIER APPLIED SCIENCE 1989, p 436.

[3]:J. Bras, K. Biyadi, M. Fagot, J.Degauque, JMMM 101,1991, 369-371

[4]:X. Xiao, K.J. Strnat, ninth international workshop on REM and their application. Bad soden, august sept $1987 \mathrm{p} 467$

[5]:J.F. Herbst, C.D. Fuerst, R.K. Mishra, J. Appl. Phys. 69(8) 15 avril $91, p 5823$. 